DOI: 10.4274/jarem.galenos.2021.52714

J Acad Res Med 2021;11(3):288-90

\title{
Anesthesia and Postoperative Analgesia for a Patient with Mungan Syndrome, Autosomal Recessive Familial Visceral Myopathy: A Case Report
}

\author{
(D) Mert Canbaz, (1) Nur Canbolat, (1) Esra Saka, (1) Mehmet Ilke Büget
}

Istanbul University, İstanbul Faculty of Medicine, Department of Anesthesiology and Reanimation, İstanbul, Turkey

Cite this article as: Canbaz M, Canbolat N, Saka E, Büget Mi. Anesthesia and Postoperative Analgesia for a Patient with Mungan Syndrome, Autosomal Recessive Familial Visceral Myopathy: A Case Report. J Acad Res Med 2021;11(3):288-90

\begin{abstract}
Mungan syndrome, a type of familial visceral myopathy, is a rare autosomal recessive genetic disorder characterized mainly by pseudo-obstruction caused by visceral myopathy. There are different components of this syndrome, such as Barret's esophagus, megaduodenum and cardiac abnormalities. Anesthetic management and procedures for postoperative analgesia are essential for patients with this syndrome to prevent complications such as ileus caused by pseudo-obstruction. This report presents our experience managing anesthesia during total hip replacement surgery performed on a 39-year-old female patient diagnosed with Mungan syndrome.

Keywords: Anesthesia, Mungan syndrome, myopathy, pseudo-obstruction, Barret's esophagus
\end{abstract}

\section{INTRODUCTION}

Familial visceral myopathies are rare genetic disorders with idiopathic chronic intestinal pseudo-obstruction syndromes (1). In these diseases, pathological findings are general degeneration and fibrotic replacement of the smooth muscles of the gastrointestinal system. In addition, the uterus and the external ophthalmoplegia of the eye may also be affected in some patients. There are many subtypes of this disease in the literature: type 1 is autosomal dominant, type 2 is autosomal recessive with ptosis and external ophthalmoplegia, and type 3 is autosomal recessive with complete gastrointestinal tract dilation (2). Mungan syndrome (MGS), an autosomal recessively inherited disorder, is familial visceral myopathies. The case reports of patients with MGS were first published in 2003 by Mungan et al. (3) Following that, the term
MGS was first described in 2007 in the literature. Genetic studies are still in progress, and linkage analysis identifies a syndromic locus on the chromosome 8q23-q24 about this syndrome (4). Its primary manifestation is chronic intestinal pseudo-obstruction caused by visceral myopathy. Gastrointestinal hypomotility, mega duodenum, and Barret's esophagus are other gastrointestinal manifestations of MGS. Patients with this syndrome may also have bilateral ptosis on the eyes, renal hypoplasia, vesicoureteral reflux, and cardiac abnormalities such as membranous ventricular septal defect, pulmonary valve regurgitation, tricuspid valve regurgitation, pulmonary valve stenosis, and supravalvular pulmonary stenosis. Management of patients with visceral myopathies is challenging due to the high risks of gastrointestinal and cardiovascular complications. As far as we know, there is no

ORCID IDs of the authors: M.C. 0000-0003-3489-6944; N.C. 0000-0003-1490-3027; E.S. 0000-0002-7170-3577; M.I.B. 0000-0002-8321-6346. 
case report regarding anesthetic management of familial visceral myopathies. This report presents a successful case of anesthetic management of a patient who had MGS operated for coxarthrosis by the orthopedic surgery department.

\section{CASE PRESENTATION}

A 39-year-old female patient was scheduled for total hip replacement surgery for coxarthrosis. Written informed consent was obtained from the patient. Her medical history revealed glaucoma, transient ischemic attack, and MGS components, including Barrett's esophagus confirmed by endoscopy and vesicourethral reflux. She had many constipation-diarrhea periods and acute gastroenteritis attacks because of the pseudoobstructive symptoms of the syndrome. Her family history was positive for familial visceral myopathy. One of her brothers died because of ileus caused by MGS, and the other brother died when he was a child for an unknown reason. Her physical examination was normal, and she had no constipation and diarrhea complaints in the preoperative period. An echocardiogram determined degenerative changes on the mitral and aortic valves. Routine non-invasive monitoring was performed, including noninvasive blood pressure, heart rate, pulse oximetry $\left(\mathrm{SpO}_{2}\right)$, electrocardiography, and temperature $\left({ }^{\circ} \mathrm{C}\right)$ monitoring. An epidural catheter was uneventfully placed through the L3-L4 vertebral space for postoperative analgesia. Anesthesia was induced with midazolam (0.05 mg/kg), fentanyl (1 mcg/ $\mathrm{kg})$, propofol $(2-2.5 \mathrm{mg} /$ $\mathrm{kg})$ and vecuronium $(0.1 \mathrm{mg} / \mathrm{kg})$. The trachea was intubated, and anesthesia was maintained with total intravenous anesthesia (TIVA) with propofol (0.1-0.2 mg/kg/min) and remifentanil (0.25-0.5 mcg/ $\mathrm{kg} / \mathrm{min}$ ) infusion and oxygen/air for the duration of the procedure. Cefazolin $(25 \mathrm{mg} / \mathrm{kg})$, ranitidine $(1 \mathrm{mg} / \mathrm{kg})$ and metoclopramide (10 mg) were administered intravenously. The bispectral index and invasive blood pressure were also monitored. The patient had episodes of hypertension whose systolic blood pressure reached $180 \mathrm{mmHg}$, and we used a bolus of $1 \mathrm{mg} / \mathrm{kg}$ followed by an esmolol infusion of $0.15 \mathrm{mg} / \mathrm{kg} / \mathrm{min}$. The intraoperative blood loss was $500 \mathrm{~mL}$, and the patient received $50 \mathrm{~mL} / \mathrm{kg}$ crystalloid solution during surgery. There was no need for blood and blood products. The duration of the operation was three hours. The trachea was extubated, and she was taken to the intensive care unit (ICU) and was discharged one day later. After the test dose (3cc of $2 \%$ lidocaine) was administered, $0.125 \%$ bupivacaine was administered as an epidural infusion for 48 hours at a rate of $3 \mathrm{cc} / \mathrm{h}$. She had no pseudo-obstructive symptoms after the operation, and her bowel movements were normal. The patient had an uncomplicated surgery and an uneventful hospital stay.

\section{DISCUSSION}

Patients with MGS may have different symptoms in a wide range of severity. While some of these patients show no symptoms throughout their lifetime, some of them can be taken to operation for exploratory laparotomy for suspected mechanical obstruction, and some of them even die because of ileus. The physician needs to be careful and consider postoperative gastrointestinal complications such as constipation and ileus (2). Consumption of fewer opioid drugs for postoperative analgesia with multimodal analgesia methods and early mobilization are essential interventions to prevent pseudo-obstructive symptoms. In our case, we offered combined spinal-epidural anesthesia to the patient, but she refused to have surgery under regional anesthesia. She just accepted an epidural catheter for postoperative analgesia., So, we preferred to place the epidural catheter to relieve the patient's postoperative pain, reduce opioid consumption, and early mobilization. Patients with MGS have decreased lower esophageal sphincter tone for visceral myopathy, so some precautions, including pharmacologic agents, should be taken to prevent aspiration prophylaxis (3). Hypertension was probably a coincidental finding for our patient, but it may also be a component of the syndrome in the future. Hypertensive episodes constituted a risky situation in terms of intraoperative bleeding, so we used esmolol, the short-acting beta-blocker infusion, to overcome the intraoperative hypertension problem. The surgery was performed in the lateral decubitus position, and supports were placed on the back for positioning. Considering the possibility of displacement of the epidural catheter due to the supports placed and the risk of increased blockage or spinal injection in the procedure performed under general anesthesia, we did not prefer epidural infusion in our patient for blood pressure and pain management. Effective fluid management is crucial to prevent postoperative cardiac and pulmonary complications as MGS also has cardiac components. We used arterial pulse contour analysis to determine the patient's volume status. To achieve goal-directed fluid therapy, we set our threshold limits of pulse pressure and stroke volume variation as $12 \%$. We preferred to use TIVA in our patient, for the possible relationship between MGS and malignant hyperthermia has not yet been rule out in the literature. She was transferred to the ICU for follow-up in malignant hyperthermia risk and to monitor the return of bowel movements.

MGS is composed of different components, and its primary manifestation is gastrointestinal symptoms related to familial visceral myopathy and pseudo-obstruction. In addition, there are other symptoms such as Barret's esophagus, cardiac abnormalities, and vesicourethral reflux (3). Therefore, in anesthesia and postoperative pain management of patients with MGS, each component of this syndrome must be considered to prevent postoperative complications by utilizing some methods such as multimodal analgesia, early mobilization, and goal-directed fluid therapy. Furthermore, dynamic monitoring methods such as pulse control analysis should be considered to evaluate patients' volume status and avoid adverse consequences of cardiac pathologies.

Informed Consent: Written informed consent was obtained from the patient.

Peer-review: Externally and internally peer-reviewed.

Author Contributions: Surgical and Medical Practices - M.C., E.S.; Data Collection and/or Processing - M.C.; Analysis and/or Interpretation - N.C., M.I.B.; Literature Search - M.C., N.C.; Writing - M.C., N.C. 
Conflict of Interest: The authors have no conflict of interest to declare.

Financial Disclosure: The authors declared that this study has received no financial support.

\section{REFERENCES}

1. Anuras S, Mitros FA, Nowak TV, lonasescu VV, Gurll NJ, Christensen J, et al. A familial visceral myopathy with external ophthalmoplegia and autosomal recessive transmission. Gastroenterology 1983; 84: 346-53.

2. Schuffler MD. Chronic intestinal pseudo-obstruction. In: Feldman M, Friedman LS, Sleisenger MH, editors. Gastrointestinal and Liver Disease. Philadelphia: Saunders; 2002. p. 2140-50.
3. Mungan Z, Akyüz F, Bugra Z, Yönall O, Oztürk S, Acar A, et al. Familial visceral myopathy with pseudo-obstruction, megaduodenum, Barrett's esophagus, and cardiac abnormalities. Am J Gastroenterol 2003; 98: 2556-60.

4. Deglincerti A, De Giorgio R, Cefle K, Devoto M, Pippucci T, Castegnaro $\mathrm{G}$, et al. A novel locus for syndromic chronic idiopathic intestinal pseudoobstruction maps to chromosome 8q23-q24. Eur J Hum Genet 2007; 15: 889-97. 